Prof. Bruhns, and Strasburg by Prof. Winnecke on the 17 th and 18 th respectively, gives the following elements:-

Perihelion Passage, September 6.4104 Greenwich M.T.

$$
\begin{aligned}
& \text { Longitude of the Perihelion ... Ii } 477^{\prime \prime} \text { ) True equinox, } \\
& \text { Inclination to Ascending Node... } 25145 \text { 50 Sept. } 16 . \\
& \text { Low Perihelion Distance } \cdots \quad \cdots \quad 78 \quad 3558 \\
& \text { Motion-retrograde. }
\end{aligned}
$$

The middle observation is represented with errors of $+7^{\prime \prime}$ in longitude and $-2^{\prime \prime}$ in latitude.

A few computed positions are subjoined, but they are to be regarded only as rough ones:-

$$
\begin{aligned}
& \text { At rzh. G.M.T. R.A. N.P.D. Distance from }
\end{aligned}
$$

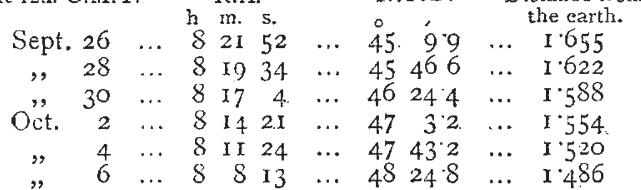

According to the above orbit the comet will remain visible for many weeks, approaching the earth, though ieceding from the sun, as it descends to the node; the intensity of light, however, is not likely to much exceed twice its actual amount. The elements do not present a striking similarity to those of any comet previcusly computed.

It is the fourth comet newly discovered in the present year, the others having been found ( $\mathrm{I}$ ) by M. Borrelly, February $8 ;(2)$ by Prof. Winnecke, April 5 ; and (3) by Mr. Swift on April I I. D'Arrest's comet of short period,

\begin{tabular}{|c|c|c|c|c|c|c|c|c|}
\hline \multirow[b]{2}{*}{ Sept. } & \multirow[b]{2}{*}{29} & & $\begin{array}{l}A t \\
\mathrm{R} A \\
\text { h. } \mathrm{m} .\end{array}$ & $\begin{array}{l}\text { Par } \\
\text { s. }\end{array}$ & is $N$ & N.P.D. & \multicolumn{2}{|c|}{$\begin{array}{l}\text { Distance from } \\
\text { the earth. }\end{array}$} \\
\hline & & $\cdots$ & $45 \mathrm{I}$ & 2 & $\cdots$ & $89.56 \cdot 4$ & $\ldots$ & 1425 \\
\hline Oct. & 3 & $\ldots$ & $45 I$ & 12 & $\ldots$ & 90326 & $\ldots$ & $1 \cdot 416$ \\
\hline " & 7 & $\ldots$ & 450 & 44 & $\ldots$ & $91 \quad 8.5$ & $\cdots$ & 1.409 \\
\hline y & II & $\ldots$ & 449 & $4 I$ & $\therefore$ & 91435 & $\ldots$ & I. 403 \\
\hline " & 15 & $\ldots$ & $44^{8}$ & 2 & $\cdots$ & $92 \quad 173$ & $\cdots$ & $1 \cdot 398$ \\
\hline
\end{tabular}
which has been observed on its fourth return, is to be added to these, and as this comet is still in a position to be observed with our larger instruments a few places are subjoined :--

\section{FAMINES AND SHIPWRECKS}

THE following letter from Dr. Balfour Stewart appeared in the Times of Saturday last :-

I have read with much interest your various articles on Dr. Hunter's researches into the Madras rainfall and the possible connection of famines with sun-spots, and I perceive from the letter in your columns of yesterday by Mr. Henry Jeula, of Lloyd's, that he has found most shipwrecks during periods of maximum solar activity.

I consider it a fortunate thing for science that the physics of the sun are now judged of sufficient importance to occupy the attention of the leading journal, inasmuch as the duty of the man of science is rather quietly to continue investigating than to endeavour to force prominently before the public the results of his work.

It has been recognised now for several years that in this particular case of shipwreck as in others the study of solar physics must ultimately lead to results of national importance. In illustration of this I may quote from a lecture delivered by $\mathrm{Mr}$. Lockyer in October, 1872 ("Solar Physics," p. 423) :-

"Mr. Meldrum, a distinguished meteorologist, who lives, not in the temperate zones of the earth, where the meteorological conditions are irregular, but in the torrid zone, where regular meteorological phenomena, and among them cyclones, abound, tells us that it is no longer correct to merely associate cyclones with the tropics. He tells us that the whole question of cyclones is a question of solar activity, and that if we write down in one column the number of cyclones in any given year and in another column the number of sunspots in any given year, there will be a strict relation between them-many sun-spots, many hurricanes; few sun-spots, few hurricanes. Only this morning I have received a letter from Dr. Stewart, who tells me that Mr. Meldrum has since found that what is true of the storms which devastate the Indian Ocean is true of the storms which devastate the West Indies; and, on referring to the storms of the Indian Ocean, Mr. Meldrum points out that at those years where we have been quietly mapping the sun-spot maxima the harbours were filled with wrecks, vessels coming in disabled from every part of the great Indian Ocean. Now that surely is something worth considering, because, if we can manage to get at these things, to associate them in some way with solar activity, so that there can be no mistake about it, the power of prediction-that power which would be the most useful one in meteorology, if we could only get at it-would be within cur grasp."

I will, with your permission, make a few remarks on the present position of the problem and on the scientific policy for the future which this position naturally suggests. In the first place, what are the facts? Without the sun, the atmosphere of our earth would be as dead and inactive as a cotton mill without fre in its boiler. As in the case of such a mill, the work done will depend upon the strength of the furnace fire, so that if the fire be variable the work will vary with it. As regards our earth, we know, to begin with, of two distinct periods of meteorological variation. The one of these is due to the change of apparent position of the sun in the heavens caused by the earth's rotation, and its length is one day. The other is due to a similar change caused by the earth's revolution, and its length is one year. If the sun were intrinsically constant we should not be justified in looking for any other variation (unless we attribute some infiuence to the moon); but if the sun be variable in its power we are led to look for a corresponding variation in terrestrial activity. Now we know that the sun is a variable factor. At certain periods his disc is absolutely free from spots, while at others it is studded with these curious objects. On these last occasions we have other lines of evidence, which lead us to believe in the intense activity of the sun, or, in the forcible language once used by the late Sir J. Herschel to myself, the solar pot seems then to be boiling very rapidly. If we are to reason by analogy at all, the terrestrial pot must follow the solar one, and occurrences denoting a deficiency of energy, such as periodical famines, depending on local failures of vegetable food, will be grouped round years of deficient solar activity, while other occurrences, depending on great energy, such as cyclones and shipwrecks, will rather follow the periods of maximum solar activity. I have taken two instances (famines and cyclones), but I might have taken others of a more recondite nature, such as the phenomena of terrestrial magnetism, concerning which I believe I am justified in stating that those who work at this branch of science are satisfied that a decided effect has been traced to the variability of the sun as a cause.

It is quite right that men of science who are pursuing other branches of inquiry, that statesmen who have to utilise the teachings of science for the benefit of nations, and, permit me to add, editors of powerful journals like your own, should wish that the proof might be of a more absolutely convincing kind than that which has satisfied the workers that they are on the right way. At present the problem has not been pursued on a sufficiently large scale or in a sufficient number of places. In fine, if the attack is to be continued, the skirmishers should give way to heavy guns, and these should be brought to bear without delay now that the point of attack is known.

There are, of course, two ways of treating the problem. The evidence may be pronounced insufficient and the 
workers stopped, when the research will gradually die out from want of support. On the other hand, if, as you, Sir, admit, we are on a track of a discovery which will in time enable us to foretell the cycle of droughts, public opinion should demand that the investigation be prosecuted with redoubled vigour and under better conditions.

My object in writing this letter is to express a hope that your powerful influence may not be wanting in furtherance of a branch of inquiry from which I, as an individual worker in it, am undoubtedly of opinion that the greatest national benefit must in time arise. If forewarned be forearmed, then such a research will ultimately conduce to the saving of life both at times of maximum and minimum sun-spot frequency.

Owens College, Manchester, September 20

\section{PROF. ADAMS ON LEVERRIER'S PLANETARY THEORIES}

THE following admirable statement concerning Le1 verrier's more recent work was made on the occasion Society, in February, last year, to him by Prof. J. C. Adams, the president. It will be read with a mournful interest at the present time :-

It is not many years since our medal was awarded to M. Leverrier for his theories and tables of the four planets nearest the sun, viz., Mercury, Venus, the Earth, and Mars. Long before this he had been occupied with the larger planets, but before proceeding further with their theories he found it necessary to establish on solid foundations the theory of the motion of the earth, on which all the rest depend, and this again naturally led him to investigate the theories of the three nearer planets which, with the earth, constitute the inferior portion of the plinetary system.

By the comparison of these theories with observations, II. Leverrier was led to two interestirg results. He found that in order to bring the theories of Mercury and Mars into accordance with observation, it was neces sary and sufficient to increase the secular motion of the perihelion of Mercury, and also the secular motion of the perihelion of Mars.

Hence M. Leverrier inferred that there existed on the one hand, in the neighbourhood of Mercury, and on the other, in the neighbourhood of Mars, sensible quantities of matter the action of which had not been taken into account.

This conclusion has been verified with respect to Mars. The matter which had not been considered, turns out to belong to the earth itself, the mass of which had been taken too small, having been derived from too small a value of the solar parallax. A similar increase of the mass of the earth is indicated by the theory of Venus, and a corresponding increase of the solar parallax is likewise derived from the lunar equation in the motion of the sun.

With respect to Mercury, a similar verification has not yet taken place, but the theory of the planet has been established with so much care, and the transits of the planet across the sun furnish such accurate observations, as to leave no doubt of the reality of the phenomenon in question; and the only way of accounting for it appears to be to suppose, with M. Leverrier, the existence of several minute planets, or of a certain quantity of diffused matter circulating about the sun within the orbit of Mercury.

The results which $M$. Leverrier had thus obtained from his researches on the motions of the inferior planets added to the interest with which he now entered upon similar researches on the system of the four great planets which are the most distant from the sun. Such researches might furnish information respecting matter hitherto un. known existing in the neighbourhood of these planets.
Possibly they might afford indications of the existence of a planet beyond Neptune, and at any rate they would provide materials which would facilitate future discoveries.

As I shall have occasion to explain later on, the theories of the mutual disturbances of the larger planets are far longer and more complicated than those of the smaller, so that all that $M$. Leverrier had yet done might be almost regarded as merely a prelude to what still remained to be done. Increased difficulties, however, far from deterring, seemed rather to stimulate him to greater exertions.

On May 20, I872, M. Leverrier presented to the Academy an elaborate memoir, containing the first part of his researches on the theories of the four superior planets, Jupiter, Saturn, Uranus, and Neptune. This memoir contains an investigation of the disturbances which each of these planets suffers from the action of the remaining three. Throughout this investigation the development of the disturbing function, as well as that of the inequalities of the elements is given in an algebraical form, in which everything which varies with the time is represented by a general symbol, so that the expressions obtained hold good for any time whatever. Thus the eccentricities and inclinations, the longitudes of the perihelion and of the nodes are all left in the condition of variables. The mean parts of the major axes, which suffer no secular variations, are alone treated as given numbers.

At the end of the résumé of the contents of this memoir, given in the Comptes Rentus, M. Leverrier lays down the following almost appalling programme of the work still remaining to be done.

It would be necessary, he says, -

I. To calculate the formulie, and to reduce them into provisional tables.

2. To collect all the exact observations of the four planets, and to discuss them afresh, in order to refer their positions to ore and the same system of co-ordinates.

3. By means of the provisional tables, to calculate the apparent positions of the planets for the epochs of the observations.

4. To compare the observed with the calculated positions, to deduce the corrections of the elliptic elements of the four planets, and to examine whether the agreement is then perfect.

5. In the contrary case, to find the causes of the discrepancy between theory and observation.

Extensive as is this programme, it has already been completely carried out as regards the planets Jupiter and Saturn, and partly so as regards Uranus and Neptune.

Having received from the Academy the most effectual encouragement to pursue his researches, M. Leverrier lost no time in bringing them gradually to completion, so that they might become available for practical use.

Accordingly, on August 26, 1872 , he presented to the Academy a memoir containing a complete determination of the mutual disturbances of Jupiter and Saturn, and thus serving as a base for the theories of both these planets, which are closely connected with each other.

Again, on November I I, 1872, he presented his determination of the secular variations of the elements of the orbits of the four planets, Jupiter, Saturn, Uranus, and Neptune. These variations are mutually dependent on each other, and must be treated simultaneously. Their determination consequently involves the solution of sixteen differential equations, which are very complicated in form, and can only be integrated by repeated approximation.

This part of the work forms a necessary preliminary to the treatment of the theory of any one of these planets in particular.

On March 17, 1873, M. Leverrier presented to the Academy the complete theory of Jupiter ; and on July I4 in the same year he followed it up by the complete theory of Saturn. 